\title{
Kearns-Sayre syndrome with optic nerve atrophy phenotype: A possible biological and clinical concurrence of two mutations?
}

\author{
Agostino Berio, ${ }^{1}$ Gian Luigi Mariottini, ${ }^{2}$ Marco Frascio, ${ }^{3}$ Attilia Piazzi ${ }^{1}$ \\ ${ }^{1}$ Department of Neurosciences, Rehabilitation, Ophthalmology, Genetics and Mother-Child Sciences (DINOGMI), \\ University of Genova, Italy; ${ }^{2}$ Retired from University of Genova, Italy, Research Fellow at Department of Earth, \\ Environment and Life Sciences (DISTAV); ${ }^{3}$ Department of Surgical Sciences and Integrated Diagnostics (DISC), \\ University of Genova, Italy
}

\begin{abstract}
The authors report about the association of progressive external ophthalmoplegia, atypical pigmentary retinopathy, ataxia phenotype with onset in first months of life (Kearns-Sayre syndrome) and with optic nerve atrophy and deafness. The localization of retinal lesions was coincident with that reported by multifunctional electroretinogram (mfERG) in OPA 1 mutation. The authors hypothesize that Kearns-Sayre mitochondrial mutation may be associated with OPA 1 missense mutation, with worsening of symptomatology, as occurs in the reported case. The prolonged rehabilitation and treatment with coenzyme Q10 for many years gave positive results, with amelioration of ophthalmoplegia, stopping of aggravation of retinal damage and optic nerve atrophy, maintaining of vision some meters away, possibility of socialization and proprioceptive ability amelioration.
\end{abstract}

Correspondence: Agostino Berio, Department of Neurosciences, Rehabilitation, Ophthalmology, Genetics and Mother-Child Sciences (DINOGMI), University of Genova, Italy.

E-mail: Agostinoberio@ospedale-gaslini.ge.it

Gian Luigi Mariottini, retired from University of Genova, Italy; Research Fellow at Department of Earth, Environment and Life Sciences (DISTAV).

E-mail: Gian.Luigi.Mariottini@unige.it

Key words: Chronic progressive external ophthalmoplegia; KearnsSayre syndrome; Coenzyme Q10 therapy;optic nerve atrophy; OPA 1 mutation; coenzyme Q10.

Conflict of interest: The authors declare no conflict of interest.

Received for publication: 1 December 2021.

Revision received: 27 January 2022.

Accepted for publication: 30 January 2022.

${ }^{\circ}$ Copyright: the Author(s), 2022

Licensee PAGEPress, Italy

Journal of Biological Research 2022; 95:10308

doi:10.4081/jbr.2022.10308

This article is distributed under the terms of the Creative Commons Attribution Noncommercial License (by-nc 4.0) which permits any noncommercial use, distribution, and reproduction in any medium, provided the original author(s) and source are credited.

\section{Introduction}

Recently, important results both in the biological and medical field have been achieved about the knowledge of Chronic Progressive External Ophthalmoplegia (CPEO), a mitochondrial respiratory chain disease presenting multiorgan involvement, ${ }^{1}$ but limited outcomes have been assessed for Kearns-Sayre Syndrome (KSS), ${ }^{2}$ a variant of CPEO, associated with optical atrophy. ${ }^{3-10}$

This work aimed to report the possible pathogenetic mechanism of the disease based on personal observations, and to outline the positive results obtained with a Q10 prolonged therapy, ${ }^{11}$ associated with rehabilitation and physiotherapy.

\section{Materials and Methods}

Clinical chemistry and hematology investigations included full blood counts, serum levels of lactic and pyruvic acid, alkaline phosphatase, lactate dehydrogenase, gamma-glutamyltransferase, Creatine Kinase (CK), which were performed by common laboratory methods. For neurophysiology, motor nerve conduction studies were performed in median, ulnar, peroneal, and tibial nerves using surface electrodes for recording and stimulation; the assessed parameters were M-wave amplitude, distal latencies, conduction velocity, F-Waves minimal latency. Electromyography (EMG) was performed in muscle spontaneous activity during relaxation. Conventional parameters of individual Motor Units' Potentials (MUPs) were assessed through visual assessment.

The pattern of maximal voluntary contraction was assessed.

Muscle biopsy was obtained from quadriceps femoris muscle by surgical biopsy. The samples were stained with hematoxylin and eosin techniques.

Muscle samples were frozen in liquid nitrogen and stained for ATP-ase, NADH-tetrazoliumreductase, phosphorylase, acid phosphatase by Gomori trichrome staining method, PAS for glycogen, and oil red for lipids were utilized.

Visual Evoked Potentials (VEPs) were performed by electrical apparatus; the method is based on the stimulation of visual receptors inducing potentials in the cortical area.

Electroretinogram (ERG), namely the method for assessing the activity potentials of the external retinal layer induced by light stimulation as flash or pattern, and mfERG, the method evaluating pre-ganglionic retinal function using multifocal electroretinography and localization of lesion areas, were utilized. 
Psychomotor Development was assessed according to Wechsler Intelligence Scale for Children (WISC).

The exploration of brainstem channels by the click of $120 \mathrm{~dB}$ SPL-150 dB SPL (90 dB HL-100 dB HL) was performed to assess Brainstem Electric Acoustic Response.

Conditioned Orientation Reflex audiometry (COR; cochlear response) in free field was assessed by recording the reactions to all frequencies.

\section{Results}

The propositus, a male of unrelated parents, was born at term (weight: $3900 \mathrm{~g}$ ) after pathological pregnancy with gestosis, and cesarean section. Transaminase, aldolase, CPK, LDH after strength were normal in the mother.

At birth, he showed slight asphyxia. No other cases of neurological, myo- or ophthalmological diseases were reported in the family.

At birth, the patient showed strabismus, deep hypotonia, froglike posture, and had no head control. During the first months of life, he showed slowing in neurophysiological steps with episodes of regression. Physiotherapy was performed for muscle hypotonia of limbs and head.

Aged 1 year, 9 months, ocular consultation showed strabismus in the right eye, reduced ocular movements in all directions, mainly in up (Progressive External Ophthalmoplegia - PEO); at ophthalmoscopy, papillae appeared very pale with net margins. At 2 years of age, the patient underwent surgery for strabismus with a 6 $\mathrm{mm}$ resection of the medial rectus muscle.

VEPs showed bilateral reduced voltage (flash and pattern) suggesting abnormalities in the stretch from the retina to the visual cortex.

At 2 years, general examination confirmed the previous data, and demonstrated frontal bossing, facial asymmetry (right $>$ left), strabismus in the right eye, antimongoloid palpebral slant, malar hypoplasia, grossly formed ears, conchae with hypoplastic lobule, flat nasal root, muscle hypotonia and hypotrophy in all limbs, mainly in superior cingulum; osteotendinous and Achilles reflexes were not evocable. The patient showed frequent dystonic and athetoid movements; he couldn't sit, assume an erect position, or walk. He spoke only 4-5 words, but mimic and gestural expressivity was present. WISC was 54. Laboratory tests demonstrated high values of CPK, GOT, LDH and aldolase, as occurs in myopathy (Table 1); routine hematological tests were normal. Muscle biopsy of quadriceps femoris (hematoxylin-eosin stain) showed normal morphology of fibers without degenerative or necrotic phenomena; neither nuclear nor connective abnormalities were emphasized. Gomori stain (NAD-tetrazolium reductase, ATP-ase, PAS) showed well-differentiated muscle fibers, with the conserved distribution.

Electrical $\mathrm{V}$ waves responses were present in the acoustic brainstem (40 dB HL), demonstrating not deep hypoacusis. CPEO was diagnosed.

At 2 years, 2 months of age, ophthalmological consultation showed scanty ocular motility in both eyes; papillae appeared very pale in both eyes at ophthalmoscopy. Lenghtened focal patches of choroid-retinal atrophy with an hyperpigmentation center was present bilaterally in the inferior nasal quadrant. Pigmentary retinopathy was diagnosed. VEPs (flash and pattern) showed reduced voltage bilaterally (optic subatrophy). Ataxia was present.

At 2 years, 10 months of age, the conditions of the patient were unmodified; CPK was high (230-308 IU/L), as LDH (510-966 IU/L), and lactacidemia: $30 \mathrm{mg} / \mathrm{dL}$ (normal value $<18$ ).

The urinary amino and organic acid elimination pattern was normal (Table 2).

Aged 3 years, 9 months, physiatry consultation demonstrated the presence of movement pattern at limbs, but many dystonic interferences gave them functionally anarchic. Physiotherapy was indicated and executed.

Ophthalmology showed the absence of spontaneous or reflex movements of ocular bulbs in all directions; strabismus was observed in both eyes and the vision was reduced; CPEO associated to optic nerve sub-atrophy was diagnosed. Language structures were absent.

CPK was constantly high, while LDH was occasionally (Table 1). The physiotherapy was continued with slow improvement of postural and motor activities.

At 5 years of life, height was $109 \mathrm{~cm}$ ( $50^{\text {th }}$ percentile), weight $18.1 \mathrm{Kg}\left(25-50^{\text {th }}\right.$ percentile $)$; physiatry consultation showed that the patient was able to assume erect position and move with bimanual support and ataxic gait; he presented frequent dystonia, and the language was hypo-structured. Proprioceptive areas were defective.

Ophthalmologic consultation showed bilateral ophthalmoplegia, normal external membranes of ocular bulbs, normochoric pupils, normreagent to photomotor stimuli. Papillae were white with net margins: arteries were constricted (optic nerve subatrophy). Bilaterally, at 4 hours in the nasal inferior quadrant, white chorioretinal patches of atrophy surrounded by a pigmentation halo were observed.

Visual loss: the patient grasped objects at $70 \mathrm{~cm}$. Computed Tomography (CT) was normal.

Table 1. Laboratory tests at different patient's ages. NV: Normal values; nd: not detected.

\begin{tabular}{|c|c|c|c|c|c|c|}
\hline \multirow[t]{2}{*}{ Parameter } & \multicolumn{6}{|c|}{ Age } \\
\hline & 2 years & $\begin{array}{l}2 \text { years, } \\
10 \text { months }\end{array}$ & $\begin{array}{l}3 \text { years, } \\
9 \text { months }\end{array}$ & 5 years & $\begin{array}{l}5 \text { years, } \\
9 \text { months }\end{array}$ & $\begin{array}{l}6 \text { years, } \\
9 \text { months }\end{array}$ \\
\hline CPK IU/L (NV <150) & $274 ; 108 ; 145 ; 210$ & $230 ; 308 ; 235$ & 254 & 230 & 213 & $231 ; 274$ \\
\hline LDH IU/L (NV <612) & $83 ; 282$ & $510 ; 966 ; 177$ & 562 & 477 & $450 ; 572$ & 506 \\
\hline GOT IU/L $(\mathrm{NV}<40)$ & 46 & nd & nd & nd & 31 & nd \\
\hline GPT IU/L $(\mathrm{NV}<40)$ & 12 & nd & nd & nd & 17 & 7.8 \\
\hline Aldolase IU/L (NV <10) & 10.6 & nd & nd & nd & $5.3 ; 11.2$ & nd \\
\hline Lactacidemia mg/dL (NV 6-16) & nd & 30 & $\mathrm{~N}$ & nd & nd & 19 \\
\hline $\begin{array}{l}\text { Pyruvicemia mg/dL(NV 0.3-0.5) } \\
\text { L/P NV < } 20\end{array}$ & nd & nd & $\mathrm{N}$ & nd & nd & $\begin{array}{c}0.2 \\
2 \mathrm{~L} / \mathrm{P}=86\end{array}$ \\
\hline
\end{tabular}


Physiatry consultation did not show retractions or articular limitations; frequent dystonic movements disturbed the action. The utilization of a special walker was indicated.

Acoustic Brainstem Electrical Responses (control) presented bilaterally $\mathrm{V}$ waves to $110 \mathrm{dbSPL}$, meaning mid-severe hypoacusis. The patient was prothesized.

At 6 years, 9 months of age, the patient showed ataxic type walking after active aid, as well as abnormalities in EMG and in motor neural fibers velocity (Table 3 ). From this age to 9 years, 9 months of age, the patient complained of bilateral ptosis, ophthalmoplegia, atypical pigmentary chorioretinal degeneration, muscle weakness, ataxia, hyporeflexia and optic nerve subatrophy; KSS was diagnosed. The values of blood lactate, pyruvate, and serum creatinine phosphokinase (Table 1) were in the range $19-30 \mathrm{mg} / \mathrm{dL}$ (normal values: 6-16), $0.22 \mathrm{mg} / \mathrm{dL}$ (normal values:0.3-0.5), $274 \mathrm{IU}$ (normal values: up to 150 ), respectively. $\mathrm{L} / \mathrm{P}$ rate $=86$. EMG was of myopathic type (Table 3). Electrocardiogram (ECG), electroencephalogram (EEG), and Magnetic Resonance Imaging (MRI) were normal.

Based on the criteria of Rowland, ${ }^{10}$ a diagnosis of KSS, a CPEO-variant with optic nerve sub-atrophy, was made.

We assumed that an overload of metabolites (lactate) precedes the defect in energy metabolism and a chronic deficiency of Coenzyme Q, as demonstrated in tissues, ${ }^{11}$ as a pathogenetic mechanism. To lower the high lactate levels, we administered a diet with $7.5 \mathrm{~g} / \mathrm{Kg}$ /day of carbohydrates; minimal dietary allowance consisted mainly of carbohydrates with low glycemic index, ${ }^{12}$ to prevent diabetes, frequent in KSS. Fat intake was $3.2 \mathrm{~g} / \mathrm{Kg}$ /day.

After 3 months, coenzyme Q10 (2,3 dimethoxy-5-methyl 1,4 benzoquinone) ring, with a side chain of 10 isoprenoid units (30 $\mathrm{mg}$ /day) was begun; the diet was not changed.

After 6 months the choreiform movements in part disappeared, bilateral ptosis improved, and eye movements were present with only mild movements reduction; fasting lactate was $6.6 \mathrm{mg} / \mathrm{dL} .{ }^{9}$

The treatment was maintained in the subsequent months and the dosage was adequate to age.

At 11 years, 9 months of age, Conditioned orientation reflex audiometry (COR) on the free field (to electrical responses) showed bilateral neurosensorial hypoacusis for all tones of middle intensity. The stapedial reflex was absent bilaterally. Compliance pick dPA: right: -30 ; left -15 .

Possible otospongiosis or early stapes anchylosis pattern with neurosensorial hypoacusis was hypothesized. The prosthesis did not cause distortion.
The general dietary conditions and coenzyme Q10 therapy were maintained with good results.

In the subsequent months, the patient was treated with continuative movement, physiotherapy and rehabilitative treatment for motility and acquired the competence to walk with support. The logopedic treatment caused amelioration of verbal expressivity, with the utilization of more words. Peritonitis consequent to appendicitis was surgically treated with recovery.

At 12 years, 6 months of age, ophthalmology demonstrated normal eye anterior segment and pallor of papillae with normal vasa. Clear vision at 3-4 meters. ERG showed a normal pattern. Irregular morphology of VEPs pattern with reduced amplitude and raised latency was observed. Aminoacids in plasma and urine were normal (Table 4).

In subsequent years, the patient continued, with intervals, the therapy with Coenzyme Q associated with vitamin B1, B12, and carnitine. A low intake of carbohydrates with a high glycemic index ${ }^{12}$ was administered.

The patient was educated in a special school and completed the

Table 2. Output of amino acids, organic acids in urine showed by High Voltage Electrophoresis/Chromatography at 2 years of age. ,,$++++++=$ intensity of stain on paper of electrophoresis.

\begin{tabular}{lc} 
Amino acids & $\begin{array}{c}\text { Result } \\
\text { (normal amino acids pattern) }\end{array}$ \\
Lysine & ++ \\
Histidine & ++ \\
\hline Metylhistidine & + \\
Glycine & +++ \\
\hline Alanine & ++ \\
Serine & + \\
\hline Threonine & ++ \\
Glutamic acid & ++ \\
\hline Proline & + \\
Hydroxyproline & + \\
\hline Aspartic acid & + \\
Amino acids in plasma & Normal \\
(chromatography; Scriver method) & \\
\hline Organic aciduria & Normal
\end{tabular}

Table 3. Patient's neurophysiology evaluations at 6 years, 9 months of age.

Muscle/nerve tests Results

\section{EMG (left anterior tibialis muscle) \\ EMG (left deltoid muscle)}

MUPS of normal amplitude and duration

Reduced duration of some MUPS

Intermediate-interferential state of middle amplitude at the medial-reflex contractions

Maximal conduction velocity of motor neural fibers (left peroneal nerve) $\quad 44.9 \mathrm{~m} / \mathrm{sec}$ (low-normal value)

from the head of the fibula to ankle

Evoked potential latency from left Extensor Digitorum Brevis (EDB) $\quad 2.3 \mathrm{~m} / \mathrm{sec}$; normal amplitude and morphology of evoked potentials by stimulation of ankle

Maximal conduction velocity of motor nerve fibers (left median nerve) from elbow to wrist

Evoked potentials latency from left opponens pollicis muscle by stimulation at wrist
$44.3 \mathrm{~m} / \mathrm{sec}$ (low-normal value)

$2.8 \mathrm{~m} / \mathrm{sec}$ normal evoked potentials amplitude and morphology 
course at 15 years of age. Some results were obtained and signaled by teachers: positive results for the psychomotor area (proprioceptive, tactile, thermal discrimination), non-verbal communication, oculo-manual coordination, socio-affective area with special interest for musical activities.

The results were strongly disturbed by dystonia and athetoidchorea-type movements. The lactic acid in blood varied from 4.5 to $30 \mathrm{mg} / \mathrm{L}$, pyruvic acid from 0.52 to $0.55 \mathrm{mg} / \mathrm{L} . \mathrm{CPK} 213 \mathrm{U} / \mathrm{L}, \mathrm{LDH}$ 572 (normal values $<619 \mathrm{IU} / \mathrm{L}$ ). Echocardiography was normal.

At 18 years of life dorso-lumbar scoliosis was diagnosed, which was surgically treated without problems with good results.

At 20 years, the ophthalmologic consultation showed pallor of the optical papillae with net margins; macular reflex was normal. Ocular bulbs were in a primitive position; their movements, even though reduced, were possible in all directions. Bilateral palpebral ptosis, more evident on the left, was observed (Figure 1).

Facial dysmorphism with grossly formed conchae, mandibular prognathism, flat philtrum, narrow cupid's arch, large nasal root, high forehead were present at 23 years of age (last control). Frequent chorea and athetoid movements and hypoacusis (prothesized) were observed.

During the period of observation, the coenzyme Q10 dosage varied from 30 to $45 \mathrm{mg} / \mathrm{die}$, about age.

\section{Discussion}

In our case, the unexplained combination of multisystem symptoms (namely psychomotor, neurologic, muscular, ophthalmologic, otologic, dysmorphic), the progressive clinical course

Table 4. Amino acids in plasma $(\mu \mathrm{Mol} / \mathrm{L})$ and urine as recorded by Amino-analyzer Carlo Erba (Milano) 3A 30. Nd: not detected.

\begin{tabular}{|c|c|c|}
\hline & $\begin{array}{c}\text { Amino acids in plasma } \\
(\mathrm{\mu Mol} / \mathrm{L})\end{array}$ & $\begin{array}{l}\text { Amino acids in urine } \\
\text { ( } \mathrm{MMol} / \mathrm{mg} \text { creatinine) }\end{array}$ \\
\hline Taurine & 5 & 142 \\
\hline Glycine & 231 & 97 \\
\hline Isoleucine & 49 & nd \\
\hline Arginine & 91 & nd \\
\hline Phenylalanine/Tyrosine & 0.91 & nd \\
\hline Aspartic acid & 4 & nd \\
\hline Alanine & 337 & 34 \\
\hline Leucine & 105 & nd \\
\hline Threonine & 116 & 17 \\
\hline Citrulline & 23 & nd \\
\hline Tyrosine & 36 & nd \\
\hline Serine & 150 & 41 \\
\hline Phenylalanine & 51 & nd \\
\hline Ac-Glutamine & 14 & nd \\
\hline Valine & 206 & nd \\
\hline Ornithine & 46 & 5 \\
\hline Glutamine & 438 & nd \\
\hline Cystine & 50 & nd \\
\hline Lysine & 148 & 26 \\
\hline Methionine & 22 & nd \\
\hline Histidine & 93 & 149 \\
\hline
\end{tabular}

with episodes of exacerbations, ${ }^{13}$ the neonatal hypotonia, the involuntary movements, the metabolic indicators of a respiratory chain disorder (hyperlactacidemia, raised lactic/pyruvic acid rate $)^{13}$ depose for a definite diagnosis of respiratory chain disorder. ${ }^{13}$ Even if the muscular biopsy in our case was negative, the clinical and laboratory data pointed out the diagnosis of an encephalomyopathy respiratory chain disorder-related. ${ }^{1}$ In our patient, PEO and ptosis were the main symptoms, cardinal signs of the CPEO Phenotype due to mitochondrial disease with single or multiple deletions, ${ }^{7}$ isolated or with multisystem involvement. PEO is the hallmark of multiple mtDNA deletions. ${ }^{4}$ With PEO, a generalized myopathy, signaled by frog-like posture, hypotonia, constantly raised CPK, and sometimes LDH, MUPS of reduced duration in deltoid muscle were present.

In patients with $\mathrm{PEO}$, the molecular defect may include largescale mtDNA rearrangements, tRNA mutation or a single mtDNA deletion. ${ }^{4}$ In other patients PEO was associated with a mutation in a nuclear gene with consequent multiple mtDNA deletions, because nuclear DNA controls mtDNA, and DNA mutation induce mtDNA instability with errors in replication. In other cases, nuclear DNA mutation may induce quantitative loss of mtDNA (depletion syndrome). As mitochondria are ubiquitarian organelles, mtDNA mutation causes a widespread distribution of pathogenic mtDNA deletions, mainly in muscle, heart, and brain (post-mitotic tissues) with multisystem involvement. ${ }^{4}$

PEO may be sporadic, but frequently it is familial, transmitted by Mendelian inheritance with dominant transmission (ADPEO) or recessive (ARPEO), late-onset, with a single species of deleted mtDNA.

Mutations of nuclear genes, frequently $A N T I$, Twinkle(C 10 Orf 2), POLG, which may be associated with dominant PEO, 6,14 are responsible for multiple mtDNA deletions. These conditions differ clinically from our case due to the presence of cardiopathy or cardiomyopathy with late-onset. ${ }^{14,15}$

PEO with multiple mtDNA deletions is frequently associated with mutations of polymerase gamma $(P O L G)$, a gene responsible for mtDNAreplication. ${ }^{15}$

PEO, hypogonadism, parkinsonism, premature menopause, $P O L G$ mutation and mtDNA deletions were reported in a family and thought to be due to a co-segregation of parkinsonism disease and POLG. ${ }^{14,15}$

Our case differs from these cases because extrapyramidal symptoms were different from those present in Parkinson's disease (tremor, rigidity, unilateral bradykinesia). ${ }^{15}$ PEO with proximal muscle abnormalities and additional symptoms (spinocerebellar ataxia, dysarthria, peripheral neuropathy, or cataract) may be associated with $P O L G$ mutation, ${ }^{15}$ but these symptoms were not present in our case.

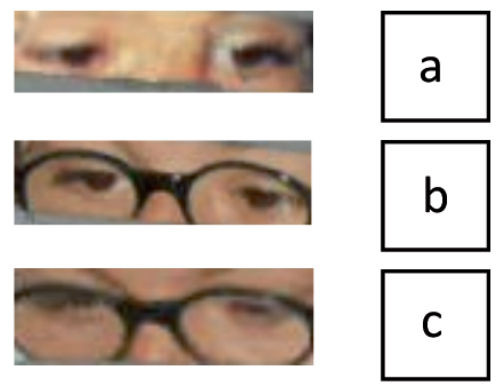

Figure 1. Direction of gaze to the left (a), to the right (b), and downwards (c). 
Mitochondrial Neurogastrointestinal Encephalomyopathy (MNGIE) syndrome due to thymidine phosphorylase nuclear mutation affecting mitochondria was not considered because gastrointestinal symptoms were not present in our patient. ${ }^{15}$

PEO with multiple mtDNA deletions may be associated with a phenotype confined to PEO with POLG mutation; ${ }^{14}$ on the contrary, our patient showed a multisystem involvement.

PEO associated with mtDNA depletion and prevalent hepatic involvement, such as in Alpers syndrome, a clinical condition due to Deoxyguanosine Kinase ( $D G U O K)$, thymidine kinase, MPV 17, were excluded in our patient by the absence of hepatic involvement and because the patients die in infancy (first months). ${ }^{16}$

Normality of aminoaciduria and organic aciduria excluded forms with Krebs cycle abnormalities and with ribonucleotide reductase deficiency. ${ }^{7}$

Contrary to genetic coenzyme Q10 defect with mental retardation, cerebellar atrophy and seizures, a disease due to a mutation of non oxidative phosphorylation nuclear-encoded mitochondrial protein, ${ }^{15}$ in our case seizures and Leigh syndrome were absent.

On the contrary, in our patient congenital strabismus, PEO, skeletal muscle involvement, raised CPK, not evocable Achilles reflex, proprioceptive disorders, low-normal motor nerve conduction velocity, interpreted as axonal neuropathy, ${ }^{17}$ motor neuron abnormalities at EMG, all depose for a PEO with multisystem disorders and muscle-nerve involvement (PEO-variant). Pigmentary retinopathy with growth abnormality observed in the patient placed PEO in the disease spectrum of $\mathrm{KSS},{ }^{18}$ a clinical syndrome characterized by onset before 20 years, pigmentary retinopathy, ophthalmoplegia and one of the following symptoms: ataxia, cardiopathy, raised protein in Central Nervous Fluid (CNF). ${ }^{5}$ The syndrome is sporadic and is due in $90 \%$ of cases to large $(1.8-8.8 \mathrm{~Kb})$ mtDNA deletions or a mtDNA replication, but also to multiple deletions or to tRNA mutation, ${ }^{19}$ a condition habitually involving other genes. Cases of superimposed symptoms with other syndromes (MERRF, MELAS), or cases due to mtDNA deletion and pyruvate-dehydrogenase complex defects were reported with combined features.

Transcription of mtDNA is polycistronic; the gene deletion could affect transcription even of genes not directly encompassed by the deletion; ${ }^{8}$ deleted mtDNA may be transcripted, but not translated. The syndrome presents a severe prognosis, with death before the third decade of life. The severity of prognosis in KSS depends on the entity and distribution of normal and mutant mtDNA, i.e. on the degree of heteroplasmy. CPEO and KSS describe different degrees of severity of the same disease, KSS showing a more severe prognosis than CPEO. 8,20

Muscle biopsy in KSS shows Ragged Red Fibers (RRF), anisometry of fibers, reduction in number and intensity of fibers cytochrome-c oxidase (COX)-positive, respiratory chain enzymes (mainly COX) deficiency. ${ }^{1,4}$ In our case, the negativity of biopsy may be due to the sample which is too small. ${ }^{1}$ The moderate visual defect is caused in KSS by pigmentary retinopathy. ${ }^{1}$ Retinal pigment derives from the neuroectoderm of the embryonic vesicles: it was thought that the primary lesion of the retinopathy in KSS resides in the pigmented cells ${ }^{21}$ and retinopathy is the expression of a distinct process of seemingly similar mutations which does not explain the mutation in that particular area. ${ }^{4}$ This suggests that the pathogenetic mechanism of the pigmented retinopathy goes beyond a simple energy defect. ${ }^{4}$ In our opinion, it is possibly connected to neural crest development. ${ }^{22}$

The pigmentary lesion in KSS affects primarily the retinal pigment epithelium; photoreceptor degeneration follows the retinal cells atrophy and pigment accumulates in the outer retina, with secondary layer degeneration. ${ }^{21}$ On ophthalmoscopy, the retinopathy in KSS involves mainly the posterior fundus and typically the central peripapillary region. ${ }^{21,23}$ The retinal atrophy, characterized by alternating pigmented and depigmented areas, is more evident in the nasal peripheral retina: the area placed on the temporal side than the macula is relatively spared. Areas of pigmentary degeneration and atrophy with small clumps are reported in the peripheral retina. $^{23}$ Diffuse depigmentation of pigment retinal epithelium with salt and pepper pattern is frequent. ${ }^{23}$ The nasal peripapillary and outer layer of the peripheral retina may be devoid of pigment, with a severe degree of atrophy of the pigment epithelium.

The optic disc is normal, rarely, pale, but normal on histology. ${ }^{21}$

On histology the retina outer layer, temporal to the optic papilla, showed an atrophic layer of photoreceptors and nuclei; Bruch's membrane was present, the peripheral temporal retina was relatively spared. If the optic nerve is normal, ganglion cells (from which optic nerve fibers originate) were relatively conserved in the inner retina as a plexiform nerve layer. ${ }^{21}$ Temporal to the macula, islands of retinal pigment epithelium are conserved surrounded by atrophy zones. Nasal retina to the optic disc showed areas of more severe atrophy, alternating with areas devoid of pigment. In the nasal peripheral layer, large aggregates of granules were observed. The choroid was atrophic. ${ }^{21}$

Similarly to our case, in a patient reported by Berenberg 23 (number 4 of cases) there were areas of pigmentary degeneration and atrophy in both retinas with small clumps at the periphery. Drachman reported two cases with KSS phenotype associated with optic nerve atrophy. ${ }^{24}$

In a case of KSS with unmeasurable visual impairment (optic nerve atrophy?) and Central Nervous System (CNS) degeneration, rods and cones layer were absent, pigment cells layer was reduced, ganglion cells were not found. ${ }^{25}$ This picture drives to a possible different mechanism for the combined association retina-optic atrophy reported in KSS by some Authors. ${ }^{21,23-25}$

The association of optic nerve atrophy with KSS is not constant. In the personal casuistry of KSS (6 patients), only one case (that here is reported) was associated with optic atrophy.

In our patient, CPEO with optic atrophy was early diagnosed and the triad of KSS at 6 years, 9 months was associated with myopathy, PEO, auditory and neuropsychological defect, chorea and athetoid movements (attributed to globus pallidus lesions ${ }^{6}$ ) and optic nerve atrophy.

In this case, the clinical and ophthalmologic picture eliminated the considerations for hereditary optic neuropathy of Leber, which presents onset in juvenile age and shows a peripapillary zone with telangiectasias, swollen disc, thick neural fiber layer, and dilated vessel around the disc.

Hereditary optic atrophy includes a group of disorders in which cell death is confined in retinal to ganglion cells, rich in mitochondria in the pre-myelinated stage, and therefore vulnerable to perturbations of mitochondria function. ${ }^{26}$ Axons damage, low voltage of VEPs, due to mitochondrial disease and frequently to OPA mutations are associated.

$O P A 1$ is the more frequently involved gene. It encodes a dynamin-related protein guanosine-triphosphatase (GTP), located in the chromosome 3q28-q29; OPA 4, with or without deafness, located in the chromosome 18q12.2-q12:3, ${ }^{3}$ and OPA 5, located in the chromosome 22q12.1-q13.126 are variants.

Additional disorders in OPA 1, such as ophthalmoplegia, deafness, and myopathy were reported. ${ }^{27}$

Optic atrophy associated with the bilateral visual failure with childhood onset, followed by deafness, PEO, myopathy, ataxia, 
sensory-motor neuropathy, and heterozygous $O P A 1$ mutation was reported in a family. In this family, some members showed multiple deletions of mtDNA in skeletal muscle, expression of mtDNA maintenance defect. Defective complex I and histochemical complex IV were shown. ${ }^{27}$

$O P A 1$ is relevant in maintaining stability, structural integrity, and oxidative phosphorylation processes of mitochondria. ${ }^{27}$ GTP targets the mitochondria; OPA 1 downregulation leads to mitochondrial membrane disruption and mitochondria apoptosis. Targeting the mitochondrial OPA 1 mutation induces secondary mtDNA defect and optical nerve atrophy by impairment of GTP protein and induction of multiple mtDNA deletions. OPA 1 mutation induces optical nerve atrophy. Being a nuclear gene, $O P A 1$ is transmitted by mendelian trait with variable penetrance (from 43 to $100 \%$ ), and dominant (DOA I) or recessive (ROA I) transmission.

Dominant OPA 1 (DOA) is characterized by optical atrophy with different severity whose features are: bilateral pallor of papillae with restriction of vasa at ophthalmoscopy, slow loss of vision and moderate loss of color perception, ${ }^{3,27}$ normal ERG pattern, pathological Multifocal Electroretinography (mfERG), ${ }^{28}$ pathological VEPS recording related to the progression of the disease. In some cases, DOA may be associated with PEO, CNS damage, ataxia, peripheral nerve motor-sensory alterations, autosomal dominant optical atrophy, and deafness (ADOAD), a condition defined as OPA 1 plus, expression of multiorgan damage due to ATP synthesis defect. ${ }^{27}$

The recent technique mfERG allows to study of the retina and optic nerve atrophy by assessing the bioelectrical responses derived from different retinal areas; the first order "kernel" of mfERG originates in the pre-ganglionic elements (photoreceptors and bipolar cells). ${ }^{28}$

Cascavilla et al. ${ }^{28}$ reported some cases of DOA studied by mfERG, in which a genotype-phenotype correlation was demonstrated, with differences in DOA haploinsufficiency (DOA H) and DOA missense mutation (DOA M).

In all DOA eye groups of patients (with both haploinsufficiency and missense), the prevalent dysfunction was found to occur mainly in the temporal inferior and the nasal superior sectors, confirming the preferential involvement of the nasal sector in which the papilla-macular bundle distributes. In OPA 1 missense mutation, mfERG showed a wide, pre-ganglionic functional defect of photoreceptors and bipolar cells due to the altered function of ganglion cells, demonstrated in temporal inferior, nasal inferior, nasal superior sectors, compared to controls, and sparing in temporal superior sector. ${ }^{28}$

The chorioretinal atrophy on the nasal inferior retinal quadrant in our case is coincidental to the dysfunction area signaled by mfERG in OPA 1 missense. The nasal area corresponds to the anatomical distribution of the papilla-macular bundle indicated by mfERG in OPA 1 missense mutation. ${ }^{28}$

Since ganglion cells altered function in OPA 1 mutation express stages of involution before degeneration, and $\mathrm{mfERG}$ correlates with retinal thickness, ${ }^{28}$ the pathologic condition leading to optic nerve atrophy and papilla-macular bundle damage was attributed to a pre-ganglionic impairment of receptors, amacrine and bipolar cells (demonstrated by mfERG) in association with a primitive ganglion cells abnormality in DOA. ${ }^{25}$

We hypothesize that in our case with normal ERG, but the low voltage of VEPs, optical papillae pallor, retinal atrophy in nasal inferior quadrant show pre-ganglionic impairment and optic nerve atrophy due to a primitive ganglion cells damage with subsequent papilla-macular bundle degeneration; the localization of pigment at 4 hours, and then in nasal inferior quadrant is coincidental with pigmented lesions reported in KSS retinal periphery.
The localization of the retinal injuries in our patient seems associate pigment abnormalities reported in KSS to the damage (papillary pallor) reported in $O P A 1$ mutation with optic nerve atrophy and draws attention to a possible coexistence of the mutations of KSS and $O P A 1$ with aggravation of retinal and optic nerve damage and contribution of retinal atrophy to KSS phenotype in our case.

An OPA 1-plus disease with multisystem involvement and optic nerve atrophy should be considered, but the pigmented retinopathy was not reported in this family of OPA 1 mutation. ${ }^{27}$

A pure optic atrophy early onset due to TMEM $126 \mathrm{~A}$ gene mutation located in chromosome $8 \mathrm{q}$ 21-q22 (OPA 6) with the recessive transmission of the congenital blindness, normal mental development, nystagmus, sensory deafness (ROA 1$)^{26,27}$ is very rare and the shown phenotype is different from the condition of our patient.

Genetic studies were not possible in our patient, due to family difficulties. The therapy with Coenzyme Q10, ${ }^{11}$ which was showed defective in muscle in some encephalomyopathies, ${ }^{29}$ was administered to the patient based on previous personal experience $^{9}$ and literature reports. ${ }^{11}$ Limited intake of carbohydrates with a high glycemic index ${ }^{12}$ was prescribed to prevent diabetes. ${ }^{29}$ This treatment was associated with Coenzyme Q10 therapy, carnitine and vitamins intake, physiotherapy, kinesitherapy, and logotherapy, and was followed (with some intervals) by the patient till to the last control.

During the first years of treatment, we observed some positive results (raised muscle straight, possibility to walk with mild support, reduction of involuntary movements, slow progression of the visual defect), which seem to confirm our diagnosis.

The patient was educated in a special State school. He acquired (at 15 years) the possibility to indicate some colors and to identify the persons at 3-4 meters; thanks to the acoustic prosthesis he showed passion for music, mainly contemporary one, showed possibility of socialization with other persons, and ameliorated proprioceptive ability. Limited results were obtained in the language with the acquisition of 5-6 words (attributed to a retarded diagnosis of deafness and of starting of logotherapy) but with acquisition of a gestural language. A limited autonomy in personal management and in the relationships with other persons was obtained. These results were confirmed by parents and teachers. Subsequently, some grade of spasticity and a dorso-lumbar scoliosis were reported and the patient at 18 years was treated by vertebral stabilization of D3-L4 and subsequent fisiokinesitherapy, with the acquisition of postural and assisted ambulation.

The lactacidemia presented oscillations, but limited increase from normal values was shown. Diabetes did not occur, and the glycemic values remained within normal limits. At 21 years, the vision permitted some socialization, and the bulbs were in the primary position with present but reduced motility in all directions.

The first results of the therapy with coenzyme $Q^{9,22}$ were confirmed in other patients with chorioretinal degeneration, CPEO, optical atrophy, deafness, and cataract, in which the defective concentration of this coenzyme was demonstrated in muscle. ${ }^{30}$ Recently, some results in the treatment of optical atrophy were reported with Idebenone, an analog of coenzyme Q10.

Beneficial effects of coenzyme Q 10 might to be due to different mechanisms, i.e. improvement of coenzyme $Q$ deficiency, increase of the membrane permeability, reduced damage by oxydants, interference with oxidative phosphorilation metabolism. ${ }^{9,29}$ This study outlines the relevance of coenzyme Q on optic atrophy associated with respiratory chain dysfunctions such as KSS associated with optic atrophy. The absence of diabetes in our case would highlight the relevance of the diet in KSS.

The difficulty to explain the pigmentary retinopathy in associ- 
ation with other symptoms in KSS was outlined. ${ }^{4}$ Based on the association of retinopathy and optic nerve atrophy in the patient, we have hypothesized the association of the deletion of KSS with $O P A 1$ mutation, with interference on retinal pigment cells and optic nerve development.

This hypothesis permitted the treatment of this condition with some positive results and possibly the nosological distinction between KSS and KSS associated with optic nerve atrophy.

This work underlines the relevance of mitochondria in KSS, as neurophthalmologic diseases, ${ }^{31}$ and the complexity of features in this syndrome. ${ }^{32,33}$

\section{References}

1. Leonard JV, Schapira AHV. Mitochondrial respiratory chain disorders II: neurodegenerative disorders and nuclear gene defects. Lancet 2000;355:389-94.

2. Kearns TP, Sayre GP. Retinitis pigmentosa, external ophthalmoplegia, and complete heart block: unusual syndrome with histologic study in one of two cases. AMA Arch Ophthalmol 1958;60:280-9.

3. Kerrison JB, Arnould VJ, Ferraz Sallum JM, et al. Genetic heterogeneity of dominant optic atrophy, Kjer type: Identification of a second locus on chromosome 18q12.2-12.3. Arch Ophthalmol 1999;117:805-10.

4. Di Mauro S, Schon EA. Mitochondrial respiratory-chain diseases. N Engl J Med 2003;348:2656-68.

5. Mishra R. Kearns Sayer Syndrome - A Case Report. J Med Res 2020;6:271-2.

6. Rahman S, Hanna MG. Diagnosis and therapy in neuromuscular disorders: diagnosis and new treatments in mitochondrial diseases. J Neurol Neurosurg Psychiatry 2009;80:943-53.

7. Schapira AH. Mitochondrial diseases. Lancet 2012;379: 1825-34.

8. Moraes CT, Di Mauro S, Zeviani M, et al. Mitochondrial DNA deletions in progressive external ophthalmoplegia and KearnsSayre syndrome. N Engl J Med 1989;320:1293-9.

9. Berio A, Piazzi A. Improvement of Kearns-Sayre syndrome with controlled carbohydrate intake and coenzyme Q10 therapy. Ophthalmologica 1994;208:342-3.

10. Rowland LP, Hays AP, Di Mauro S, et al. Diverse clinical disorders associated with abnormalities of mitochondria. In: Scarlato G, Cerri C, eds. Mitochondrial pathology in muscle diseases. Padova: Piccin, 1983:141-58.

11. Ogasahara S, Yorifuji S, Nishikawa Y, et al. Improvement of abnormal pyruvate metabolism and cardiac conduction defect with coenzyme Q10 in Kearns-Sayre syndrome. Neurology 1985;35:372-7.

12. Willett W, Manson JA, Liu S. Glycemic index, glycemic load, and risk of type 2 diabetes. Am J ClinNutr 2002;76:274S-80S.

13. Bernier FP, Boneh A, Dennett X, et al. Diagnostic criteria for respiratory chain disorders in adults and children. Neurology 2002;59:1406-11.

14. Hirano M, Di Mauro S. ANT1, Twinkle, POLG, and TP: new genes open our eyes to ophthalmoplegia. Neurology 2001; 57:2163-5.

15. Schapira AH. Mitochondrial disease. Lancet 2006;368:70-82.

16. Sarzi E, Bourdon A, Chrétien D, et al. Mitochondrial DNA depletion is a prevalent cause of multiple respiratory chain deficiency in childhood. J Pediatr 2007;150:531-4, 534.e1-6.

17. Melberg A, Lundberg PO, Henriksson KG, et al. Muscle-nerve involvement in autosomal dominant progressive external ophthalmoplegia with hypogonadism. Muscle Nerve 1996;19:751-7.

18. Pfeffer G, Sirrs S, Wade NK, et al. Multisystem disorder in late-onset chronic progressive external ophthalmoplegia. Can J Neurol Sci 2011;38:119-23.

19. Seneca S, Verhelst H, De Meirleir L, et al. A new mitochondrial point mutation in the transfer RNA (Leu) gene in a patient with a clinical phenotype resembling Kearns-Sayre syndrome. Arch Neurol 2001;58:1113-8.

20. Moraes CT, Shanske S, Tritschler HJ, et al. mtDNA depletion with variable tissue expression: a novel genetic abnormality in mitochondrial diseases. Am J Hum Genet 1991;48:492-501.

21. Eagle RCJr, Hedges TR, Yanoff M. The atypical pigmentary retinopathy of Kearns-Sayre syndrome. A light and electron microscopic study. Ophthalmology 1982;89:1433-40.

22. Berio A. Metabolic Syndromes and neural crest development. J Biol Res 2011;84:73-5.

23. Berenberg RA, Pellock JM, Di Mauro S, et al. Lumping or splitting? "Ophthalmoplegia-plus" or Kearns-Sayre syndrome? Ann Neurol 1977;1:37-54.

24. Drachman DA. Ophthalmoplegia plus. The neurodegenerative disorders associated with progressive external ophthalmoplegia. Arch Neurol 1968;18:654-74.

25. Horwitz SJ, Roessmann U. Kearns-Sayre syndrome with hypoparathyroidism. Ann Neurol 1978;3:513-8.

26. Barbet F, Hakiki S, Orssaud C, et al. A third locus for dominant optic atrophy on chromosome 22q. J Med Genet 2005;42:e1.

27. Hudson G, Amati-Bonneau P, Blakely EL, et al. Mutation of OPA1 causes dominant optic atrophy with external ophthalmoplegia, ataxia, deafness and multiple mitochondrial DNA deletions: a novel disorder of mtDNA maintenance. Brain 2008; 131:329-37.

28. Cascavilla ML, Parisi V, Triolo G, et al. Retinal dysfunction characterizes subtypes of dominant optic atrophy. Acta Ophthalmol 2018;96:e156-e163.

29. Zierz S, Jahns G, Jerusalem F. Coenzyme Q in serum and muscle of 5 patients with Kearns-Sayre syndrome and 12 patients with ophthalmoplegia plus. J Neurol 1989;236:97-101.

30. Rötig A, Appelkvist EL, Geromel V, et al. Quinone-responsive multiple respiratory-chain dysfunction due to widespread coenzyme Q10 deficiency. Lancet 2000;356:391-5.

31. Chertkof J, Hufnagel RB, Blain D, et al. Retinoschisis associated with Kearns-Sayre syndrome. Ophthalmic Genet 2020;41:497-500.

32. Pasquini L, Guarnera A, Rossi-Espagnet MC, et al. Spinal cord involvement in Kearns-Sayre syndrome: a neuroimaging study. Neuroradiology 2020;62:1315-21.

33. Tsang SH, Aycinena ARP, Sharma T. Mitochondrial Disorder: Kearns-Sayre Syndrome. Adv Exp Med Biol 2018;1085:161-2. 